\title{
MIPYME ACUÍCOLAS: EXTENSIÓN RURAL GUBERNAMENTAL, POLÍTICAS PÚBLICAS Y PROGRAMAS ACADÉMICOS UNIVERSITARIOS EN COSTA RICA ${ }^{1}$
}

\author{
Ruth Vargas-Cordero², Daniela Quirós-Arias ${ }^{3}$
}

\section{RESUMEN}

Realizar un análisis general de los planes gubernamentales y la orientación de las principales carreras dictadas por las universidades estatales, las cuales tienen por precepto dirigir esfuerzos para graduar profesionales cuya responsabilidad será desarrollar y fortalecer el sector agropecuario y afines así como la evaluación generada in situ, de los pequeños emprendimientos acuícolas, sus debilidades y potencialidades, sus productos y cómo los comercializan fue el objetivo de este trabajo. Mientras que la primera parte del binomio planes/realidad se establece a niveles jerárquicos superiores en los organismos y entidades estatales y mundiales; la recopilación de eventos en la segunda parte proviene de la estrecha relación con los actores de proyectos productivos de toda escala. De ahí que permiten concebir cómo es la estructura y los medios de financiamiento y asesoría reales que tienen las empresas para generar y procesar productos de origen acuícola. Éstos con previsiones de hasta $33 \%$ de crecimiento para el 2021 , dado que son cada vez más consumidos a nivel nacional y global. Algunas compañías de mayor capital podrían incrementar la posibilidad de hacer frente a la expansión comercial con gran inversión incluso extranjera con el ánimo de recaudar divisas que no necesariamente quedan en el país; por el contrario, las micro, pequeñas y medianas empresas agropecuarias que en Costa Rica representan un $12,70 \%$ del total de las declaradas como MIPYME, utilizan sus recursos para reinvertir como soporte económico a las familias rurales generando fuentes de empleo que alivian en parte la migración a las ciudades, sirva de ejemplo los 1. 241 pequeños emprendimientos acuícolas en Costa Rica.

Palabras clave: Microempresa, Desarrollo rural, acuicultura, pequeños emprendimientos, planes estatales, técnicos acuícolas.

\footnotetext{
${ }^{1}$ Inscrito en la Vicerrectoría de Investigación. Universidad de Costa Rica. Proyecto 739-B3-269

${ }^{2}$ Universidad de Costa Rica. Facultad de Ciencias Agroalimentarias. Centro de Investigaciones en Nutrición Animal. Autor para correspondencia ruth.vargas@ucr.ac.cr

${ }^{3}$ Universidad de Costa Rica. Estudiante de Escuela de Zootecnia. Autor para correspondencia daniqa0502@gmail.com

Recibido: 3 de agosto $2015 \quad$ Aceptado: 27 de mayo 2016
} 


\section{ABSTRACT}

Aquaculture MSME: Government rural extension, public policies and university academic programs in Costa Rica. Contrast government plans and the orientation of the major careers dictated by state universities, which have by precept to direct efforts to graduate professionals whose responsibility will be to develop and strengthen the agricultural sector and related industries, versus evaluation generated in situ, of small aquaculture enterprises, weaknesses and potential, their products and how and they market was the goal of this work. While the first part of the dichotomy plans: reality are set by senior levels in government and global agencies, event collection in the second part comes from the close relationship with the actors of productive projects of any scale. Hence allow conceive how the structure and means of financing and real advice these companies have to generate and process products of aquaculture origin. These, with estimates of up to $33 \%$ growth for 2021 ; since they are increasingly consumed nationally and globally. Large companies face huge commercial opportunity with foreign investment even with the intention of earning foreign currency do not necessarily remain in the country; by contrast, the micro, small and medium enterprises (MSME) in Costa Rica farming represent $12.70 \%$ of companies declared, MSME use their resources to reinvest and economic support to rural families generating employment sources relieving partly migration to cities, serve as an example the 1.241 small aquaculture ventures in Costa Rica.

Keywords: microbusiness, rural development, aquaculture, small enterprises, state plans, aquaculture technicians. 


\section{INTRODUCCIÓN}

Una dinámica empresarial cada vez más veloz y compleja en el escenario mundial ha convergido en los últimos años en un interés creciente por la micro, pequeña y mediana empresa (MIPYME), debido al aporte determinante de éstas para el desarrollo nacional en aspectos económicos, ambientales y sociales (Brenes, 2008) y por tanto como fuentes generadoras de empleo (Saavedra y Hernández, 2008).

En Latinoamérica las MIPYMES representan alrededor de un 99,13\% (Saavedra y Hernández, 2008) de las empresas formales. En Costa Rica se calcula que un 97,85\% de las empresas formales corresponden a esta categoría, de las cuales un $12,70 \%$ se dedican a la agricultura y pesca (MEIC, 2014). De éstas, el Instituto Costarricense de Pesca y Acuicultura (INCOPESCA 2013) reportó para 2012 un total de 1.241 proyectos acuícolas, de los cuales el $92,42 \%$ están dedicados a la producción de tilapia y trucha.

A nivel general se reconoce que estas empresas representan uno de los sectores clave para mejorar la situación de desempleo en la mayor parte de las economías a nivel mundial, además pueden tener un papel protagónico en la creación de fuentes de innovación y competitividad en nuevas actividades de producción o áreas de servicio (ONU, 2007).

Su importancia a nivel latinoamericano se ve reflejada en la investigación hecha por Saavedra y Hernández (2008), donde se expone que la proporción de microempresas es en promedio del $89,86 \%$.

Alrededor del mundo existen una gran variedad de formas de considerarlas y definirlas según las necesidad de cada país o los objetivos que se persigan, por ello se pueden manejar los argumentos más relevantes que generen determinada clasificación para que con base en ella, sean aplicadas las políticas, medidas y estrategias económicas que propicien el desarrollo de estos estratos empresariales (Saavedra y Hernández, 2008).

En Costa Rica, la clasificación de micro, pequeña y mediana empresa se da mayormente según la cantidad de personal que integra el sistema productivo, denominándose como microempresas aquellas que poseen un máximo de 15 empleados, pequeñas un máximo de 30 y medianas 100 colaboradores, siendo 
grandes compañías las que cuenten con más de 100 personas como recurso humano en sus planillas de trabajo (MEIC, 2014).

Paralelamente, la creciente demanda mundial de proteína animal ha representado un desafió especialmente en la segunda mitad del siglo $\mathrm{XX}$, presionando el potencial productivo natural de pastizales y del océano; situación que derivó en que el consumo de carne de res en 2012 fue de 63 millones de toneladas, comparativamente la de pescado fue de 66 millones; con la particularidad que esta última provino del cultivo controlado y no de la extracción (Larson y Roney, 2014).

Sobre este comportamiento FAO (2012), prevé para el año 2021 un 33\% de incremento de la actividad acuícola por sobre otros sectores pecuarios; bajo un marco en el que el tema de la seguridad alimentaria, eje de la Cumbre Mundial de Alimentación en 1996, provocó que como prioridades normativas de este organismo y a fin de cumplir con dicho mandato, la seguridad alimentaria combinase el enfoque de doble componente para combatir el hambre: agricultura y el desarrollo rural sostenibles, con programas específicamente dirigidos a incrementar el acceso directo a los alimentos para los sectores más necesitados, implementando para ello medidas como, revitalización del sector pecuario asociado a inversión en infraestructura (FAO, 2006).

No obstante si bien se atribuye relevancia al papel gubernamental en el tema de la extensión en acuicultura, FAO (2013) señala que en la mayoría de los países latinoamericanos no existe la plataforma suficiente para acompañar de forma eficaz los procesos productivos y las demandas de asistencia técnica de los productores de las MIPYME acuícolas.

En relación al componente educativo como eje central del desarrollo en una economía global, el Sistema de Educación Superior Universitaria Estatal coordinado por el Consejo Nacional de Rectores (CONARE), enlistó para el quinquenio 2011-2015 una serie de compromisos para con la sociedad costarricense en el acápite vinculación con el entorno. Entre ellos, continuar realizando acciones en torno a seguridad alimentaria, redes con organizaciones sociales y productivas de diferentes regiones y específicamente, impulsar programas y proyectos de emprendimiento, particularmente en los que se implementen instrumentos derivados de la Ley PYME y otras iniciativas de desarrollo empresarial, conjuntando fortalezas y actividades de las universidades estatales (CONARE, 2011). 
El objetivo de este trabajo es exponer la situación actual de las MIPYME que dentro de sus actividades tengan el componente acuícola, así como las medidas gubernamentales y académicas tendientes al fortalecimiento del desarrollo rural y de dicha actividad; lo anterior por cuanto estos son los sectores involucrados y coresponsables de encaminar la producción primaria y su industrialización en búsqueda de su mejoramiento.

\section{DESARROLLO TEMÁTICO}

La evaluación de los aspectos vivenciales a nivel de campo en las MIPYME con componente acuícola, derivó en entrevistas a actores involucrados en el sector público, a fin de dar soporte a las primeras conclusiones.

Con base en ello, seguidamente se hizo una evaluación exhaustiva de fuentes bibliográficas y documentación disponible en instituciones nacionales relacionadas al sector de las pequeñas empresas, al sector agropecuario y comercial, incluyendo la banca estatal; con el fin de obtener la visión que respalde el accionar presente y futuro de las MIPYME en aspectos considerados de mayor relevancia: legales-organizativos, técnico-empresariales, humanos, asesoramiento y desarrollo rural y ambientales.

Posteriormente, para completar el análisis general, se valoraron planes de estudio de carreras de universidades públicas, responsables de formar profesionales que transferirán los conocimientos técnicos que soporten un mejoramiento económico al poblador campesino sea sobre la base de las acciones del INCOPESCA, el Ministerio de Agricultura y Ganadería (MAG), el Banco Central de Costa Rica (BCCR) o cualquier entidad que procure fortalecer el desarrollo rural y la actividad acuícola en el territorio nacional.

\section{Aspectos legales - organizativos}

La normativa que da cabida al concepto de MIPYME, es el reglamento de la ley de fortalecimiento a las pequeñas y medianas empresas $\left(\mathrm{N}^{\circ}\right.$ 8262), en el Decreto Ejecutivo 37121 (MEIC, 2014). Donde se define como: unidad productiva de carácter permanente (al menos un año de permanencia en el mercado) que dispone de 
recursos físicos estables y de recursos humanos; los maneja y opera, bajo la figura de persona física o persona jurídica, en actividades industriales, comerciales o de servicios, excluyendo aquellas actividades económicas de subsistencia (Brenes, 2008).

El Instituto Costarricense de Pesca y Acuicultura (INCOPESCA, 2015) es el ente encargado de coordinar y ordenar el desarrollo de la pesca, la caza marítima, la acuicultura y la investigación. Asimismo sobre la base de criterios científicos y técnicos la conservación, el aprovechamiento y el uso sostenible de los recursos hídricos. De ahí que norma, elabora y vigila la aplicación de la legislación. Entre sus parágrafos está también la coordinación de actividades con organismos de crédito, de asistencia técnica y de cualquier otra índole que fomente el aprovechamiento pesquero, la estabilización de precios y la conservación de las especies. Bajo este marco tiene la atribución de proponer el programa nacional para el desarrollo de la pesca y la acuicultura, de conformidad con los lineamientos que se establezcan en el Plan Nacional de Desarrollo. Empero según sus políticas, en materia de investigación no se externa un programa de esta naturaleza.

A pesar de lo anterior no es sino hasta febrero de 2016, que el estado por medio del ente Rector coordinó con la Organización de la Naciones Unidas para la Alimentación y la Agricultura (FAO) y el Ministerio de Agricultura y Ganadería, el desafío de convocar al Primer Diagnóstico de la Acuicultura en Costa Rica, como el mecanismo para determinar la situación del sector y tener la visión clara de las potencialidades y retos sectoriales (Meneses, 2016).

Durante el periodo 2010-2013 el Poder Ejecutivo (MAG, 2014) impulsó dos importantes gestiones que beneficiaron la actividad agropecuaria: aprobación de la Ley 9071 de Regulaciones Especiales sobre la Aplicación de la Ley 7509 del Impuesto sobre Bienes Inmuebles, que reconoce el derecho a tasar de manera diferenciada la tierra de uso agropecuario para el pago del impuesto, además de una propuesta, sobre la Gestión Integrada del Recurso Hídrico y que contempló las preocupaciones por preservar y proteger este recurso bajo el vínculo inevitable agua-producción.

Así los hitos de evolución del Sector Agroalimentario, tuvieron legalmente asidero: en 2011 el Reglamento a la Ley de Pesca y Acuicultura. Decreto No. 36 782, la Definición de un fondo concursable y su reglamentación para la asignación de las transferencias de recursos públicos a proyectos de pequeños productores y la puesta en marcha del Plan Sectorial de Género y Juventud Rural. En 2012 se declaró de interés público y nacional según Decreto No. 36 866, la Feria del Gustico costarricense, la que ha Nutrición Animal Tropical 10(1): 45-65 ISSN: 2215-3527/ 2016 
generado ya tres ediciones. En 2013, se planteó el Plan Nacional de Desarrollo de la Pesca y Acuicultura Decreto No. 37 587-MAG; así como el Decreto No. 37 911-MAG Definición de pequeño y mediano productor agropecuario.

Para el cierre 2014 se señaló en firme la aprobación en primer debate por la Asamblea Legislativa a la Ley para la Gestión Integrada del Recurso Hídrico y la Declaratoria de zonificación para la pesca de atún en la zona económica exclusiva del Pacífico Oriental de Costa Rica.

En materia regulatoria se mejoraron (MAG, 2014) entre otros trámites: la obtención del Certificado Veterinario de Operación (CVO), el Registro de Medicamentos Veterinarios, el Registro de Alimentos para Animales y el Certificado y Registro exportación/importación; así como la verificación y aprobación sanitaria por parte de Servicio Nacional de Salud Animal (SENASA) para el punto de ingreso y salida en el des-almacenaje, exportación, tránsito nacional o internacional.

\section{Aspectos técnico-empresariales}

EI MAG (2014) apunta que el sector agroalimentario costarricense para el periodo 2010 - 2013 representó un pilar fundamental de la economía, puesto que el valor agregado agropecuario (VAA) alcanzó el 14\% del producto interno bruto, el cual incluye la agricultura, la producción animal, la pesca y la agroindustria; siendo que fueron exportados 935 productos de origen agroalimentario que generaron 4.100 millones de dólares en divisas anuales y que representaron el $38 \%$ del valor total de las exportaciones del país.

La Promotora del Comercio Exterior de Costa Rica (PROCOMER, 2013), indicó que los productos de origen acuícola han sufrido importantes cambios de 2011 a 2012 en relación a su exportación, claro ejemplo de ello el hecho de que el rubro filetes y demás carnes de pescado cuyo valor FOB pasó de 54,7 millones USD y una posición 30 a 73,8 millones, ocupando la posición 23 .

Lo anterior tuvo cabida (MAG, 2014) en términos generales, dado el fortalecimiento de circuitos cortos de comercialización de los productos internos, a partir de la inserción de los productores en el Centro Nacional de Abastecimientos y Distribución de Alimentos - Programa Integral de Mercadeo Agropecuario (Cenada-PIMA) y, los estudios para el establecimiento de dos mercados locales, uno en la Región 
Chorotega y otro en la Región Brunca. También dos acciones importantes por intermediación del Programa de Abastecimiento Institucional (PAI) y ferias del agricultor, ya que fueron fortalecidas como mercado estratégico.

Se fortaleció el sistema estadístico agropecuario, ya que en 2013 en alianza con el Instituto Nacional de Estadística y Censos de Costa Rica (INEC) y el Banco Central de Costa Rica (BCCR) se llevó a cabo la Construcción del directorio de fincas y establecimientos agropecuarios dedicados principalmente a actividades agrícolas, ganaderas, forestales y acuícolas, como una herramienta fundamental para desarrollar el sistema de encuestas trimestrales de área y producción agrícola (MAG, 2014).

\section{Aspectos humanos}

MAG (2014) advierte que el sector MIPYME generó empleo directo a 273.000 personas y se mantuvo como el segundo sector empleador. Considerando que en las MIPYME la mano de obra es familiar, el papel de la mujer tiene relevancia con respecto a otras actividades pecuarias y productivas $\mathrm{o}$ al menos ha sido abordada a mayor profundidad; sirva de ejemplo dicho papel, en el ambiente marino-costero (Aguilar y Castañeda, 2000).

\section{Aspectos asesoramiento y desarrollo rural}

EI MEIC (2014) indica que la proporción MIPYME costarricense está liderada por las microempresas que representan el $74 \%$, mientras el $12 \%$ son pequeñas y el $14 \%$ restante se compone de medianas, además señala que existe una MIPYME por kilómetro cuadrado y una unidad empresarial por cada 85 habitantes, dándose un aumento de estas desde el año 2007 en adelante.

Considerándose a la agricultura familiar (en el contexto de la producción primaria de las MIPYME), como pilar de la gestión de los territorios rurales, fue sujeta de su rescate por parte del sector (MAG, 2014), por dos vías: por una parte se transformó el Instituto de Desarrollo Agrario en Instituto de Desarrollo Rural, mediante Ley N ${ }^{\circ} 9036$, procurando que se trascendiera del modelo de asentamiento campesino a uno que contemple la participación activa de las comunidades en la gestión de sus territorio.

Por otro lado se potenció a los Consejos Territoriales de Desarrollo Rural (CTDR) para elaborar Planes Territoriales de Desarrollo Rural (PTDR) que dinamicen la inserción de 
las comunidades en los procesos de desarrollo productivo, comunal y social. Por ejemplo, bajo el marco del Programa Préstamo (1566/OC/CR) - Programa de Desarrollo Sostenible de la Cuenca Binacional del Río Sixaola, orientado a mejorar las condiciones de vida de la población del Cantón de Talamanca, se benefició a 15.420 familias. Además, se incorporaron cuatro nuevas Áreas Marinas de Pesca Responsable (AMPR).

Sirva como ejemplo lo anterior para indicar que si bien se está dando una tendencia gubernamental en miras de fortalecer el área de maricultura en las zonas marinocosteras del país, Aguilar y Castañeda (2000), advierten que [...] en el caso de los programas y proyectos de desarrollo pesquero, creados a partir de una cantidad limitada de conocimiento técnico estandarizado, tipo paquete tecnológico, que en su aplicación no reconocen las particularidades estructurales y funcionales de los ecosistemas, las relaciones sociales y medios de producción. El resultado es el fracaso de acciones poco precisas en sus fases de identificación, formulación, ejecución, seguimiento, evaluación, por lo tanto, atienden a una realidad subjetiva.

Cabe destacar también que, Costa Rica invirtió en el periodo 2010-2014 por medio del Ministerio de Agricultura y Ganadería (MAG, 2014) 8.761 millones de colones de recursos del programa de transferencias, para apoyar los procesos productivos desarrollados por organizaciones que integran a 19.038 productores y productoras y ejecutó 117 proyectos distribuidos en todo el país. Los recursos se orientaron al desarrollo de infraestructura productiva en hortalizas, apicultura, ganadería, caña de azúcar; apoyo a procesos de comercialización de café, ganadería, hortalizas, granos básicos y frutas; modernización de ferias del agricultor, infraestructura y equipamiento; comercialización y agroindustria en ganadería, granos básicos, hortalizas, lácteos, frutas, miel de abeja y chile dulce y tecnologías y sistemas de producción sostenible en café y hortalizas.

\section{Evaluación a nivel de campo}

La producción piscícola continental de las MIPYME está orientada básicamente a trucha y tilapia; esta última localizada en diversas regiones: Zona Norte, Guápiles, Guácimo, Talamanca y la región sur desde San Isidro hasta Buenos Aires de Pérez Zeledón; mientras que la trucha se produce básicamente en dos: las poblaciones circunvecinas a la carretera Interamericana Sur desde La Estrella y hasta San Gerardo 
de Dota, Potrero Grande en San Vito, Toro Amarillo y algunas empresas en la zona alta de San Carlos y Zarcero (Otárola, 2015¹).

Es importante indicar que solamente tres empresas están asociadas a la producción de tilapia a gran escala, cuyos productos son principalmente exportados y en menor medida destinados al consumo nacional; mientras que una única empresa de mediana envergadura en el contexto nacional, opera en la producción de trucha con un enfoque inverso, es decir, sus productos se consumen en mayor grado en el país (Ureña, 2013²; Víquez, 2015³).

Contrariamente, los pequeños emprendimientos cuentan con espejo de agua limitado y comúnmente el sistema de flujo es en serie, aunado a que calculan el caudal según las pulgadas de la tubería que utilizan, la que es ajustada con base en la cantidad que llega a la finca. La mayoría no cuenta con estructuras que posibiliten un óptimo recambio ni la utilización de filtros apropiados, tampoco con equipo para controlar el oxígeno.

Sus registros productivos consisten en contabilizar el gasto de alimento, el que en virtud del cambio generado en las empresas productoras de alimentos balanceados favoreció un importante avance en la alimentación, en términos de utilizar piensos apropiados según el tamaño de la boca de los animales. La Dirección de Alimentos para Animales (DAA) del Ministerio de Agricultura y Ganadería (MAG), mantiene el registro de los mismos, controlando la calidad química y microbiológica por intermedio de laboratorios y procedimientos acreditados por el Centro de Investigaciones en Nutrición Animal (CINA) de la Universidad de Costa Rica; si bien es claro que las micro y pequeñas empresas no tienen ningún poder de negociación para con la Industria de Alimentos Balanceados para Animales.

En cuanto al desdoble según talla si bien se realiza en algunos emprendimientos utilizando balanzas, la mayoría fundamenta la separación por tamaño y por experiencia práctica en términos de cuántos peces ajustan el kilogramo.

Con respecto al procesamiento del pescado, son muy pocas las empresas dedicadas a esta labor. Para tilapia, coexisten alrededor de cinco, exceptuando a la mayor empresa que si está debidamente equipada para hacer frente a la exportación

\footnotetext{
${ }^{1}$ Otárola, J. comunicación personal, 2015

${ }^{2}$ Ureña, comunicación personal, 2013

3 Víquez, comunicación personal, 2015

Nutrición Animal Tropical 10(1): 45-65 ISSN: 2215-3527/ 2016
} 
internacional, mientras que las otras operan si es del caso, básicamente con el Certificado Veterinario de Operación (CVO).

En el caso de la trucha operan alrededor de cuatro con su respectivo certificado. Todas se dedican al mercado nacional, mientras que el producto exportado producido por la única empresa catalogada en este trabajo como mediana, se procesa en una planta dedicada a la exportación de producto tanto de origen marino como continental (Víquez, 2015).

Muchas explotaciones venden su producto directamente a turistas, por lo que la matanza, limpieza y preparación no cumple necesariamente con medidas aprobadas por el Ministerio de Salud.

En relación a la asesoría que reciben específicamente del ente Rector, el país cuenta con alrededor de siete técnicos, uno destinado a investigación en pargo la mancha (Lutjanus gutattus), el resto a asesorar los emprendimientos de trucha y tilapia en todo el país. Paralelamente en la Dirección General de Extensión Rural del MAG, según sus lineamientos, los técnicos visitan una amplia gama de emprendimientos agropecuarios de todo tipo (MAG, 2016).

Las cinco formas básicas de comercializar los productos son: 1) Venta in situ en centros agro turísticos, 2) entre los mismos centros agroturísticos, 3) de productor a centros agro turísticos, 4) de productor a pescaderías y otros y 5) de productor a domicilio (MAG/PFPAS 2007, Céspedes, 20155). Comparativamente a los productos marinos, en los dulceacuícolas se da un menor grado de intermediación que se refleja en que los márgenes de comercialización oscilan entre un 50 a un $75 \%$ dependiendo del producto. Para el mercado doméstico de productos provenientes de micro y pequeñas empresas, no existe un sistema de etiquetado o certificación que permita la trazabilidad comparativamente a los productos destinados a la exportación (FAO, 2015) a diferencia de la empresa que opera a mediana escala en truchas y a gran escala en tilapia.

En la agrocadena de las MIPYME es notorio el papel de la mujer comparativamente a otros sistemas pecuarios; tanto en los aspectos productivos como de procesamiento y

\footnotetext{
${ }^{4}$ Víquez, comunicación personal, 2015

${ }^{5}$ Céspedes, comunicación personal, 2015
} 
mercadeo; asimismo la concurrencia de otros medios de producción, especialmente el agrícola, coadyuvan a mejorar el ingreso de las familias rurales (Prado, $2015^{6}$ ).

\section{Papel de entidades académico-universitarias}

Se indagaron los planes de estudio ofertados de las áreas relacionadas con el sector agropecuario y el desarrollo rural, con el fin de evidenciar alguna proyección académica para que los profesionales costarricenses puedan llegar a atender las necesidades crecientes de las micro, pequeñas y medianas empresas en las zonas rurales de nuestro país y en las que la producción acuícola tiene relevancia y son, según el estado y organismos como la FAO quienes soportarán el incremento de la producción.

El Instituto Tecnológico de Costa Rica (ITCR, 2015) si bien manifiesta que el perfil de sus graduados en varias carreras como Agronegocios podrá insertarse en diversas empresas haciendo uso de los recursos y bajo una óptica humanista, no presenta explícitamente ningún énfasis a la administración y transferencia en zonas rurales y de producción acuícola; con el inconveniente que no divulga por su página web los planes de estudio de las mismas.

La Universidad Nacional, oferta el diplomado en acuicultura, dándose un enfoque especialmente técnico en la producción de especies piscícolas, igualmente ofrece la licenciatura en Biología con énfasis en Manejo de Recursos Marinos y Dulceacuícolas pero no se visualiza la parte de extensión rural en ninguna de las mismas; mientras que en la carrera de Ingeniería Agronómica se imparte un curso de Desarrollo Agropecuario Rural. (UNA, 2015).

La Universidad Estatal a Distancia (UNED, 2015), es la institución que presenta un mayor enfoque en la administración y organización de recursos rurales, tanto en la carrera de Ingeniería Agronómica como en la de Administración de Empresas Agropecuarias, donde se imparten estos cursos; sin embargo, en general para ninguna de las dos carreras se advierte sobre algún curso específico de producción acuícola (UNED, 2015).

\footnotetext{
${ }^{6}$ Prado, comunicación personal, 2015

Nutrición Animal Tropical 10(1): 45-65 ISSN: 2215-3527/ 2016
} 
En contra parte, tanto la Universidad de Costa Rica (UCR, 2015) como la Universidad Técnica Nacional (UTN, 2015), presentan en los planes de estudio de las carreras de Ingeniería Agronómica con énfasis en Zootecnia y Producción Animal respectivamente, el curso de Producción Acuícola, siendo éste optativo en el caso de la UCR desde el nivel de bachillerato. En ambas instituciones no se da enfoque de extensión u organizaciones rurales, según lo indagado en los respectivos planes de estudio.

En el caso de la carrera de Biología de la Universidad de Costa Rica, el curso referente a la acuicultura es optativo desde el nivel de bachillerato, igualmente no se oferta ninguno que relacione al graduado con la realidad rural productiva.

En la Escuela de Economía Agrícola de la Universidad de Costa Rica y a nivel de licenciatura se oferta un curso específico de Economía Ambiental y de Recursos Renovables, siendo optativos para este mismo nivel los cursos de Evaluación social de Proyectos y Economía Agrícola; pero ninguno específico al desarrollo rural.

En materia de procesamiento y conservación de productos agropecuarios, la Escuela de Tecnología de Alimentos, de la Universidad de Costa Rica, tampoco oferta - al menos por nombre - ningún curso específico para productos acuícolas ni tampoco alguno del que se deduzca la enseñanza de cómo transmitir la base de los procesos fabriles a comunidades rurales.

\section{CONSIDERACIONES FINALES}

Es claro el papel que tienen las micro y pequeñas empresas como generadoras de recursos para las muchas familias campesinas y por ende de fuentes de trabajo con inclusión de género. Los micro emprendimientos son mucho más que las empresas con mediano y alto desarrollo técnico, por lo que confluyen mayor cantidad de personas, pero lamentablemente de zonas marginadas con menor nivel educativo y menos recursos para invertir, sumado a menor infraestructura. 
Por lo que, cualquier esfuerzo que se realice para mejorar la productividad de la actividad tendrá impacto en un alto porcentaje de emprendimientos y las relaciones sociales que de ellas deriven; hay alrededor de 1237 micro y pequeñas empresas (INCOPESCA, 2013).

Esta situación se agrava más al confrontar la realidad y las políticas en materia de investigación y aprovechamiento sostenible del recurso hídrico de origen marino, tanto en los programas del estado como de las instituciones educativas; siendo importante señalar cómo la Universidad Nacional si tiene una propuesta para el desarrollo rural por medio de un programa de investigación referido a la producción de ostras marinas; así como el Parque Marino desarrolló el protocolo para la producción de pargo la mancha con igual énfasis; aunque ambas con limitado alcance real hacia las comunidades, por la vasta insuficiencia de recursos y, la complejidad y amplitud de los estudios necesarios para producir de manera sostenible.

Con base en estos dos ejemplos de transferencia, así como los fallidos emprendimientos de los acuicultores de tilapia y trucha de agremiarse, se evidencia que fomentar la acuicultura de mar y tierra de manera sostenible e inocua en las diversas regiones económicamente más deprimidas, no podrá hacerse sin apoyo financiero a la par de capacitación y acompañamiento técnico y, descentralización de centros de acopio y redes de frío y/o procesamiento; considerando además el mejoramiento de la red de caminos y carreteras.

Aunado a lo anterior, los planes de las escuelas de formación técnica orientadas a la producción, no procuran en el graduado su inserción en la economía rural. En contraparte, la Escuela de Planificación y Promoción Social de la UNA oferta una carrera totalmente orientada a que el profesional sepa guiar a los diversos actores rurales al mejoramiento de sus comunidades, pero con un plan curricular de poco componente técnico, dada la diversidad de fuentes de producción.

Esta deficitaria relación entre los diversos saberes, puede considerarse subsanada con la creación del programa del Sistema de Educación Superior Universitaria Estatal coordinado por el Consejo Nacional de Rectores (CONARE, 2011), sin embargo en la puesta en marcha de sus proyectos regionales, la participación estudiantil es mínima.

Igual planteamiento se tiene en la Política de Estado para el Sector Agroalimentario y el Desarrollo Rural Costarricense 2010-21 que se diseñó en septiembre de 2010 (Rodríguez y Flores, 2014) que indica como objetivo: elevar las condiciones de vida e ingresos de la población vinculada al sector agroalimentario, a 
través de la promoción de la competitividad, la innovación, el desarrollo tecnológico y la gestión equilibrada de los territorios rurales, apoyada en una articulación eficiente y eficaz de la institucionalidad pública y privada (MAG, 2010). En el marco del pilar de innovación y desarrollo tecnológico, se propone impulsar un Programa de Especialización en Acuicultura, dirigido a profesionales en temas de manejo genético, enfermedades y nutrición de peces y crustáceos (Estrategia 4.2.3.2).

Desafortunadamente, siete aspectos señalados a continuación permiten dudar del éxito esperado por el Estado y su propuesta del plan de desarrollo acuícola aunado al apoyo de entidades como la FAO: PRIMERO, el número de productores que son proclives a ser asesorados bajo la figura de cooperativas en Costa Rica, no debe ser inferior a 22, situación compleja en un país, en el que si bien este modelo opera en empresas pequeñas y medianas incluso en el área agropecuaria y pesca, lo cierto es que requiere un esfuerzo de varios años para su organización y sostenimiento. Sirva de soporte a lo anteriormente señalado, el hecho de que en 1963, estuvieron activas 67 cooperativas, en 1983 este tipo de organización sirvió de apoyo a 407 empresas, cantidad que se redujo a 376 en 2012 , de las que solamente $10,3 \%$ están orientadas a la agricultura, ganadería, silvicultura y pesca (INFOCOOP, 2012). Esta situación da pie para plantear que la población en general no tiene dentro de su esquema social este arquetipo. SEGUNDO: hay pocos técnicos contratados y capacitados en materia acuícola, por lo que es evidente como se han fortalecido otras áreas agropecuarias antes que de especímenes acuáticos. TERCERO: Probablemente sus conocimientos en desarrollo rural son empíricos, puesto que su formación quizá fue tal y como actualmente se están formando quienes les reemplazarán. CUARTO: desde 2014 se cerró la posibilidad de obtener permisos para uso de agua, quizá en respuesta a que no se vislumbra un incremento en fuentes de empleo para profesionales que deberán eventualmente no solo hacer las valoraciones in situ sobre la pertinencia o no de los proyectos que están operando sin los permisos y/o por la asistencia requerida que debería dárseles paralelamente para velar que se produzca bajo criterios sostenibles. QUINTO: la investigación es incipiente y los recursos de infraestructura apenas están fortaleciéndose o creando, no solo para el mejoramiento de la competitividad en la utilización y análisis de digestibilidad de materias primas para la elaboración de alimentos sino también, del potencial del recurso animal de origen local. SEXTO: la cantidad de fincas estatales para atender la acuicultura en vez de incrementarse se redujo en la última década y cumplen un papel más de suplidoras de semilla que de centros que generen u orienten la investigación a pesar del mandato que por ley 
deben hacer. SÉPTIMO: si bien hay soportes económicos foráneos en el presente quinquenio (2011 - 2015) para potenciar el desarrollo de las MIPYME acuícolas latinoamericanas, entre ellas Costa Rica, el mismo cuenta aún con dos características con que se suscitó el apoyo desde hace 45 años; la primera que a pesar de que aún no hay investigación pertinente para un replanteamiento de las dietas y su fabricación a partir de materias primas autóctonas según se indicó, se pretende reducir la dependencia de alimentos balanceados que oferta el mercado.

Esto significa, ya planteado por MAG/GTZ (1990), que la producción a baja escala permitirá a los productores autoabastecerse, pero siempre tendrán exceso de producto, pero que se verán obligados a vender solo localmente, desestimulando el interés por el negocio y el eventual abandono. La segunda característica es que dentro de los rubros presupuestados del soporte ofrecido, un alto porcentaje se destina a la asesoría, la que además no es de origen nativo.

Este análisis tiene eco en el documento preparado por Red de Acuicultura de las Américas (2014) de la Organización de las Naciones Unidas para la Alimentación y la Agricultura (FAO), en donde se reconoce que hasta ahora esta entidad impulsó la actividad bajo un enfoque asistencialista en lugar de uno direccionado a la creación de capacidades y el desarrollo productivo en las zonas rurales, lo que inhibe su autosuficiencia.

Al estudiar en detalle los pormenores de los sustentos en el presente siglo, por una parte entre sus recomendaciones se exhorta a gobernanza multinivel y multisectorial por medio de enfoques sistémicos, políticas inclusivas y alianzas entre otras [ideas]. Pero por otra, se evidencia que el apoyo económico permanece sobre la base de emprendimientos pequeños y de autosuficiencia en el uso de materias primas para alimentos, así como la dispersión del presupuesto para pagar asesorías externas que hagan el esquema de las propuestas, su evaluación y valoración una vez desplegadas en cada país, y una vez implementadas, deban ser calificadas por los pares externos.

En relación a lo que acontece en Costa Rica y partiendo de lo anteriormente señalado, se concluye que efectivamente hay debilidades de pensamiento crítico en el estado y en las universidades públicas, por ello es evidente la desarticulación nacional (mencionada por Red de Acuicultura de las Américas (2014) de la Organización de las Naciones Unidad para la Alimentación y la Agricultura (FAO) ) en materia acuícola; se incluye aquí para dirigir esfuerzos y para remozar planes educativos que formen profesionales capacitados para transmitir conocimientos de producción acuícola y en áreas rurales.

Nutrición Animal Tropical 10(1): 45-65 ISSN: 2215-3527/ 2016 
Actividad cuyo crecimiento ha sido pronosticado a nivel mundial. Es decir no existe en el país, la concatenación requerida entre el estado, algunas investigaciones y las unidades académicas para apoyar los planes de estudio, con tal de aprovechar la oportunidad ya visualizada hace más de tres décadas, en un país en el que su principal fortaleza a largo plazo es indiscutiblemente su recurso hídrico y que, además potenciaría la pluralidad social gracias a la gran cantidad de micro y pequeñas empresas.

Coexiste además un grave problema fiscal que atenta contra el incremento de contratación de profesionales en los entes estatales de los que por una situación de inercia, se incrementan los salarios estatales. Dichos profesionales en el corto plazo deberían además contar con más y mejores conocimientos y, capacidades pertinentes, tanto en acuicultura como en la asesoría de las comunidades rurales. Tres debilidades más que se pueden citar de importancia global de la producción acuícola: la lenta respuesta que ha tenido el estado por muchos años para incrementar y descentralizar la red de frío, eliminar el subsidio de la gasolina al sector pesquero asumiendo que éste es además, un punto de desencuentro entre este sector y el acuícola. 


\section{LITERATURA CITADA}

Aguilar L., I Castañeda. 2000. Sobre marinos, marinas mares y mareas: perspectiva de género en zonas marino-costeras. Unión Mundial para la Naturaleza. Área Social y Área de Humedales y Zonas Costeras. Oficina Regional para Mesoamérica-ORMA Social Policy Program. San José Costa Rica. 269 p.

Brenes L. 2008. Observatorio: Primer diagnóstico nacional de MIPYMES. Primera Edición. Editorial de Universidad Estatal a Distancia. Costa Rica. ISSN 16593502.

CONARE (Consejo Nacional de Rectores). 2011. Oficina de Planificación de la Educación Superior. Plan Nacional de la Educación Superior Estatal Universitaria Estatal 2011-2015/Consejo Nacional de Rectores. Oficina de Planificación de la Educación Superior, Comisión de Directores de Planificación.-San José, C.R.: CONARE-OPES, 2011. 175p.; 28cm.- (OPES; no.19-2010).

Escuela de Administración de Empresas. 2015. Plan de Estudios. Universidad Estatal a Distancia. Disponible en http://www.uned.ac.cr/carreras-uned. (Consultado 21 Julio 2015).

Escuela de Biología. 2015. Plan de Estudios. Universidad de Costa Rica. Disponible en http://www.biologia.ucr.ac.cr/. (Consultado 21 Julio 2015).

Escuela de Ciencias Biológicas. 2015. Plan de Estudios. Universidad Nacional Disponible en http://www.biologia.una.ac.cr/. (Consultado 21 Julio 2015).

Escuela de Economía Agrícola. 2015. Plan de Estudios. Universidad de Costa Rica Disponible en http://economia.ucr.ac.cr/sites/default/files/files/plan_2330301.pdf. (Consultado 21 Julio 2015).

Escuela de Ingeniería Agronómica. 2015. Plan de Estudios. Universidad Estatal a Distancia. Disponible en http://www.uned.ac.cr/carreras-uned. (Consultado 21 Julio 2015). 
Escuela de Planificación y Promoción Social. 2015. Plan de Estudios. Universidad Nacional. Disponible en http://www.epps.una.ac.cr/. (Consultado 27 Julio 2015).

Escuela de Producción Animal. 2015. Universidad Técnica Nacional. Disponible en http://www.utn.ac.cr/. (Consultado 21 Julio 2015).

Escuela de Tecnología de Alimentos. 2015. Plan de Estudios. Universidad de Costa Rica. Disponible en: http://www.ta.ucr.ac.cr/. (Consultado 27 Julio 2015).

Escuela de Zootecnia. 2015. Plan de Estudios. Universidad de Costa Rica. Disponible en http://www.zootecnia.ucr.ac.cr/. (Consultado 21 Julio 2015).

FAO (Organización de las Naciones Unidas para la Alimentación y la Agricultura). 2006. Informe de Políticas. Seguridad alimentaria. No. 2. Disponible en ftp://ftp.fao.org/es/ESA/policybriefs/pb_02_es.pdf. (Consultado 22 Julio 2015).

FAO (Organización de las Naciones Unidas para la Alimentación y la Agricultura). 2012. El estado mundial de la pesca y la acuicultura 2012. Disponible en: ftp://ftp.fao.org/FI/brochure/SOFIA/2012/spanish_flyer.pdf. (Consultado 21 Julio 2015).

FAO (Organización de las Naciones Unidas para la Alimentación y la Agricultura). 2013. Diagnóstico de la Acuicultura de Recursos Limitados (AREL) y de la Acuicultura de la Micro y Pequeña Empresa (AMYPE) en América Latina. Serie Acuicultura en América. $N^{\circ}$ 7. p22.

FAO (Organización de las Naciones Unidas para la Alimentación y la Agricultura). 2015. Departamento de Pesca y Acuicultura. Visión general del sector acuícola nacional. Costa Rica. Características, estructura y recursos del sector. Disponible en http://www.fao.org/fishery/countrysector/nasocostarica/es. (Consultado 27 Julio 2015). 
INCOPESCA. 2015. Sitio web del Instituto Costarricense de Pesca y Acuicultura. Disponible en https://www.incopesca.go.cr/acuicultura/acuicultura_cr.html. (Consultado 21 Julio 2015).

INFOCOOP (Instituto Nacional de Fomento Cooperativo). 2012. Programa Estado de la Nación. Síntesis IV Censo Nacional Cooperativo 2012. -1ra.ed. - San José Costa Rica. 130 p. ISBN-978-9968-859-18-9

TEC (Instituto Tecnológico de Costa Rica). 2015. Carreras. Disponible en http://www.tec.ac.cr/. (Consultado 21 Julio 2015).

Larson J., Roney M. 2014. Producción de pescado de cultivo supera la producción de carne de res. Industria acuícola, acuacultura y negocios de México. Vol. 10 No 2. 29-31 p.

MAG (Ministerio de Agricultura y Ganadería). 2016. Sitio web de información disponible en: http://www.mag.go.cr/ (Consultado 22 de Abril 2016).

MAG (Ministerio de Agricultura y Ganadería). 2014. Memoria de gestión del sector agroalimentario 2010-2014. -- San José, C.R.: MAG/Sector Agroalimentario, p164. ISBN 978-9968-877-63-3.

MAG (Ministerio de Agricultura y Ganadería). 2007. Marco del Programa de Fomento de la Producción Agropecuaria sostenible. Convenio 1436/OC-CR-BID. Plan Estratégico de la Cadena Productiva de Acuicultura. Período: 2008-2010.

MAG/GTZ (Ministerio de Agricultura y Ganadería/Agencia de Cooperación Técnica Alemana). 1990. Valoraciones y argumentos para una nueva orientación del desarrollo de la acuacultura en la Zona Atlántica (Costa Rica). Fomento de la Acuacultura.PN 85.2039.7.p 38.

MEIC (Ministerio de Economía, Industria y Comercio). 2014. Estado de la situación de las MIPYMES en Costa Rica. Primer informe de sistemas de indicadores MIPYME. p 22-23.

Meneses, G. 2016. Convocatoria a taller de diagnóstico de la acuicultura en Costa Rica. PESJ-050-2016.Instituto Nacional de Acuicultura y Pesca. San José, Costa Rica. 
ONU (Organización de la Naciones Unidas). 2007. El desarrollo de las pymes mediante el uso de tecnologías de la información y de las comunicaciones (tic): las opciones para América Latina y el Caribe. Boletín de facilitación del comercio y el transporte en América Latina y el Caribe. Boletín $N^{\circ} 254$. Disponible en: www.cepal.org/transporte/noticias/bolfall/1/32061/FAL254e.pdf. (Consultado 5 Mayo. 2015).

PROCOMER (Promotora de Comercio Exterior). 2012. Estadísticas de Comercio Exterior de Costa Rica. 2012. Dirección de Inteligencia Comercial. Calderón. J.C. San José, C.R. 245p. ISSN: 2215-2334.

Rodríguez A., Flores A. 2014. Acuicultura de pequeña escala y recursos limitados en América Latina y el Caribe. Hacia un enfoque integral de políticas públicas. Red de Acuicultura de las Américas. Organización de la Naciones Unidas para la alimentación y la Agricultura. Santiago de Chile. E-ISBN 978-92-5-308169-1.

Saavedra M., Hernández Y. 2008. Caracterización e importancia de las MIPYMES en Latinoamérica: Un estudio comparativo. Revista Actualidad Contable FACES. 11 (17): 122-134. 